\title{
NÚMERO REPRODUTIVO DA COVID-19 E O RELAXAMENTO DO DISTANCIAMENTO SOCIAL NO BRASIL
}

\section{ARTIGO ORIGINAL}

AKHAVAN, André Amin Martin ${ }^{1}$

AKHAVAN, Dariush ${ }^{2}$

AKHAVAN, André Amin Martin. AKHAVAN, Dariush. Número reprodutivo da COVID19 e o relaxamento do distanciamento social no Brasil. Revista Científica Multidisciplinar Núcleo do Conhecimento. Ano 05, Ed. 10, Vol. 07, pp. 129-137. Outubro de 2020. ISSN: 2448-0959, Link de acesso: https://www.nucleodoconhecimento.com.br/saude/numero-reprodutivo

\section{RESUMO}

A chegada da pandemia da COVID-19 no Brasil foi seguida pela adoção abrupta de medidas de distanciamento social, reduzindo radicalmente a circulação de indivíduos nas áreas urbanas brasileiras. O relaxamento progressivo de tais medidas, curiosamente, não gerou um aumento do número reprodutivo efetivo $\left(R_{e}\right)$ da COVID19. Nesse estudo, descrevemos a relação temporal entre o $R_{e}$ da COVID-19 no Brasil e a mobilidade urbana brasileira entre fevereiro e agosto de 2020 a partir de dados do Relatório de Mobilidade da Comunidade da Google e do Situation Report for COVID19: Brasil do Imperial College London, demonstrando uma queda inicial da média de mobilidade urbana brasileira de $50-60 \%$ em relação aos valores anteriores à pandemia, acompanhado também por uma queda do $R_{e}$. Formulamos, então, hipóteses que poderiam explicar a relativa estabilidade do $R_{e}$ observada ao longo dos meses em um contexto de aumento progressivo da circulação em áreas urbanas brasileiras, utilizando o modelo epidemiológico tricompartimental SIR. Sugerimos que

\footnotetext{
${ }^{1}$ Graduação em Medicina pela Universidade de Brasília.

${ }^{2}$ Médico Intensivista. 
a redução da transmissibilidade do vírus, possivelmente em decorrência de estratégias como o uso de máscaras e a higiene respiratória, junto com uma redução da proporção de suscetíveis para a doença, decorrente de uma possível imunidade natural, ainda mal caracterizada e controversa na literatura, são fatores que provavelmente justificam a estabilidade do $R_{e}$ no contexto de relaxamento do distanciamento social no Brasil.

Palavras-chave: COVID-19, coronavirus, epidemiologia, distanciamento social.

\section{INTRODUÇÃO}

A pandemia do novo coronavírus tem causado mudanças abruptas nas dinâmicas populacionais ao redor do mundo. O vírus SARS-COV-2, agente etiológico da COVID19, transmitido por gotículas respiratórias, é um coronavírus identificado pela primeira vez em 2019 na região de Wuhan da China, causador de um surto de pneumonias de agente etiológico inicialmente desconhecido. (WIERSINGA et. al., 2019) Múltiplas estratégias têm sido adotadas para a contenção da transmissão viral, incluindo medidas de higiene respiratória, baixo limiar para testagem e isolamento de casos suspeitos, busca ativa de contactantes e medidas de distanciamento social. $\mathrm{O}$ fechamento de escolas, lojas e academias, apelos para evitar aglomerações e a suspensão de procedimentos e consultas médicas de caráter eletivo foram responsáveis pela drástica redução na circulação de indivíduos dentro das cidades brasileiras observada a partir de março de 2020. (BRAZIL AUGUST, 2020) Com o progressivo relaxamento das medidas que promoveram o distanciamento social, seria intuitivo esperar um aumento do número reprodutivo efetivo $\left(R_{e}\right)$. Entretanto, por motivos pouco esclarecidos, tal efeito não foi observado. (SITUATION REPORT, 2020) Nesse estudo, apresentamos a relação temporal entre o distanciamento social e o $R_{e}$ no Brasil entre fevereiro e agosto de 2020, e formulamos hipóteses, com base no modelo epidemiológico SIR, do mecanismo por trás das tendências observadas. 


\section{METODOLOGIA}

Dados sobre a circulação de indivíduos dentro das cidades brasileiras entre 15 de fevereiro e 28 de agosto de 2020 foram obtidos através do Relatório de Mobilidade da Comunidade da Google de 1 de setembro de 2020. (BRAZIL AUGUST, 2020) relatório obtém dados anônimos e agregados a partir do uso de produtos e aplicativos da Google. Tal relatório quantifica diariamente as tendências de mobilidade em comparação ao valor basal prévio à pandemia, dividindo a mobilidade urbana em seis categorias: varejo e lazer, mercados e farmácias, parques, estações de transporte público, locais de trabalho e residencial. A partir da média aritmética entre os valores referentes às primeiras cinco categorias, obtivemos a média da mobilidade urbana (MMU).

O número reprodutivo da COVID-19 no Brasil ao longo do tempo foi obtido pelo Situation Report for COVID-19: Brasil de 1 de setembro de 2020 do Imperial College London (SITUATION REPORT, 2020). A formulação de hipóteses para justificar a variação do $R_{e}$ em função da tendência de mobilidade urbana foi baseada no modelo epidemiológico SIR, um modelo tricompartimental (suscetíveis, infecciosos e recuperados) estabelecido na literatura que permite modelar a dinâmica de propagação de doenças infecciosas.

\section{RESULTADOS}

A média da mobilidade urbana (MMU) sofreu queda brusca em meados de março, alcançando a máxima redução em torno da última semana de março, entre - $-50 \%$ e $60 \%$. Desde então, houve um progressivo aumento desse indicador de forma relativamente linear, alcançando $-10 \%$ até o fim de agosto.

Entre as seis categorias da mobilidade urbana, conforme o gráfico 1, observou-se queda mais significativa em meados de março da atividade em parques, estações de transporte público e varejo e lazer, atingindo valores entre -60\% e - $-80 \%$. Quedas menos significativas foram observadas em locais de trabalho, de $-40 \%$ a $-60 \%$, e em mercados e farmácias, de $-20 \%$ a $-40 \%$. Ao longo dos meses até o fim de agosto 
ocorreu aumento progressivo nesses cinco indicadores. Houve aumento apenas no componente residencial, de $+20 \%$, coincidindo com as quedas nos outros componentes em meados de março, com progressiva redução, atingindo $+10 \%$ até o fim de agosto.

Gráfico 1: Variação da mobilidade urbana brasileira entre fevereiro e agosto de 2020

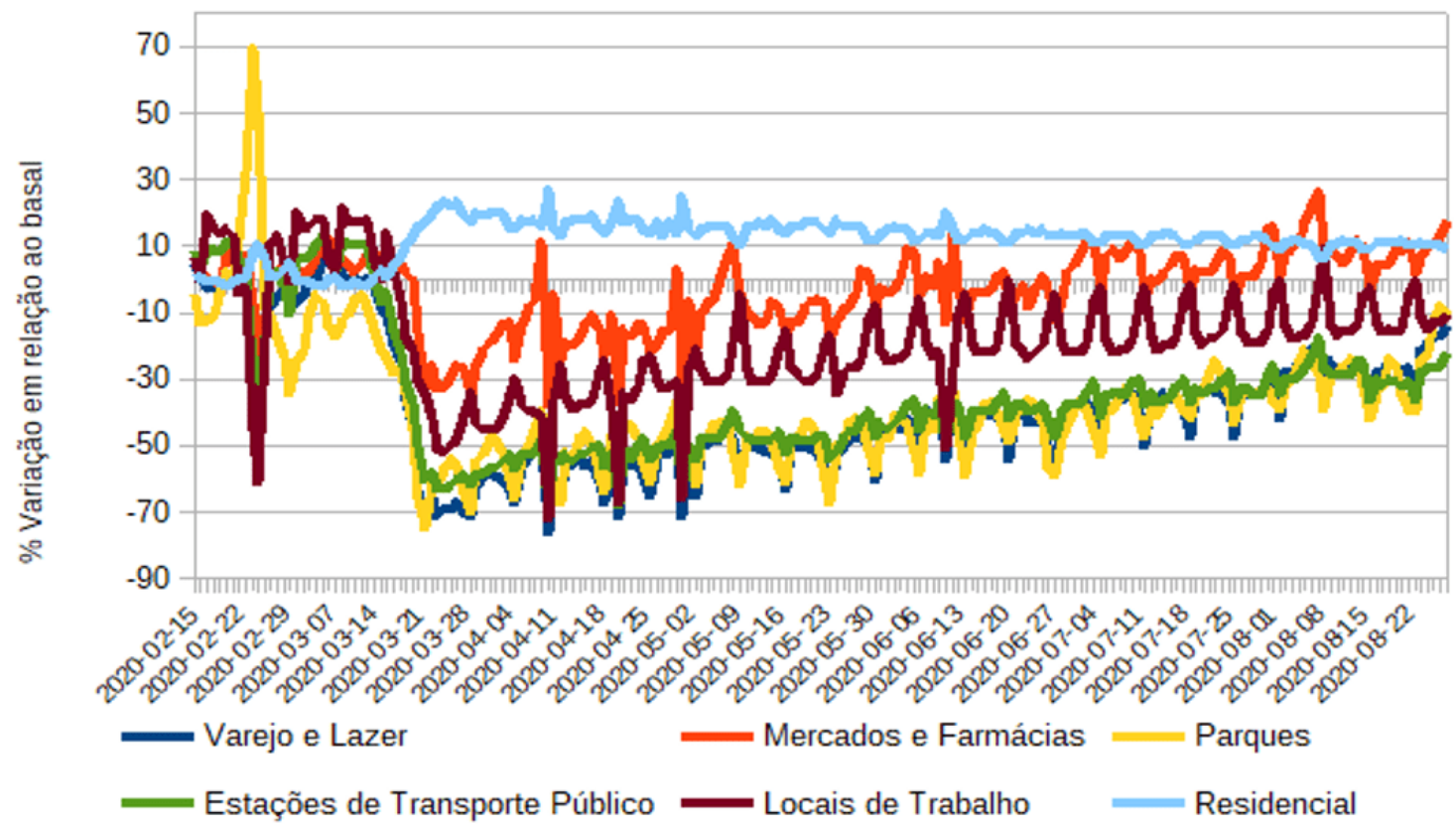

Fonte: BRAZIL AUGUST (2020)

O $R_{e}$ da COVID-19 no Brasil observado no início do período estudado, conforme o gráfico 2, se mantinha entre 3,5 e 4, com queda súbita coincidindo com a diminuição da MMU observada em meados de março, se estabilizando em torno de 1 entre maio e agosto. 
Gráfico 2: Relação temporal entre a média da mobilidade urbana brasileira e o número reprodutivo efetivo da COVID-19 no Brasil

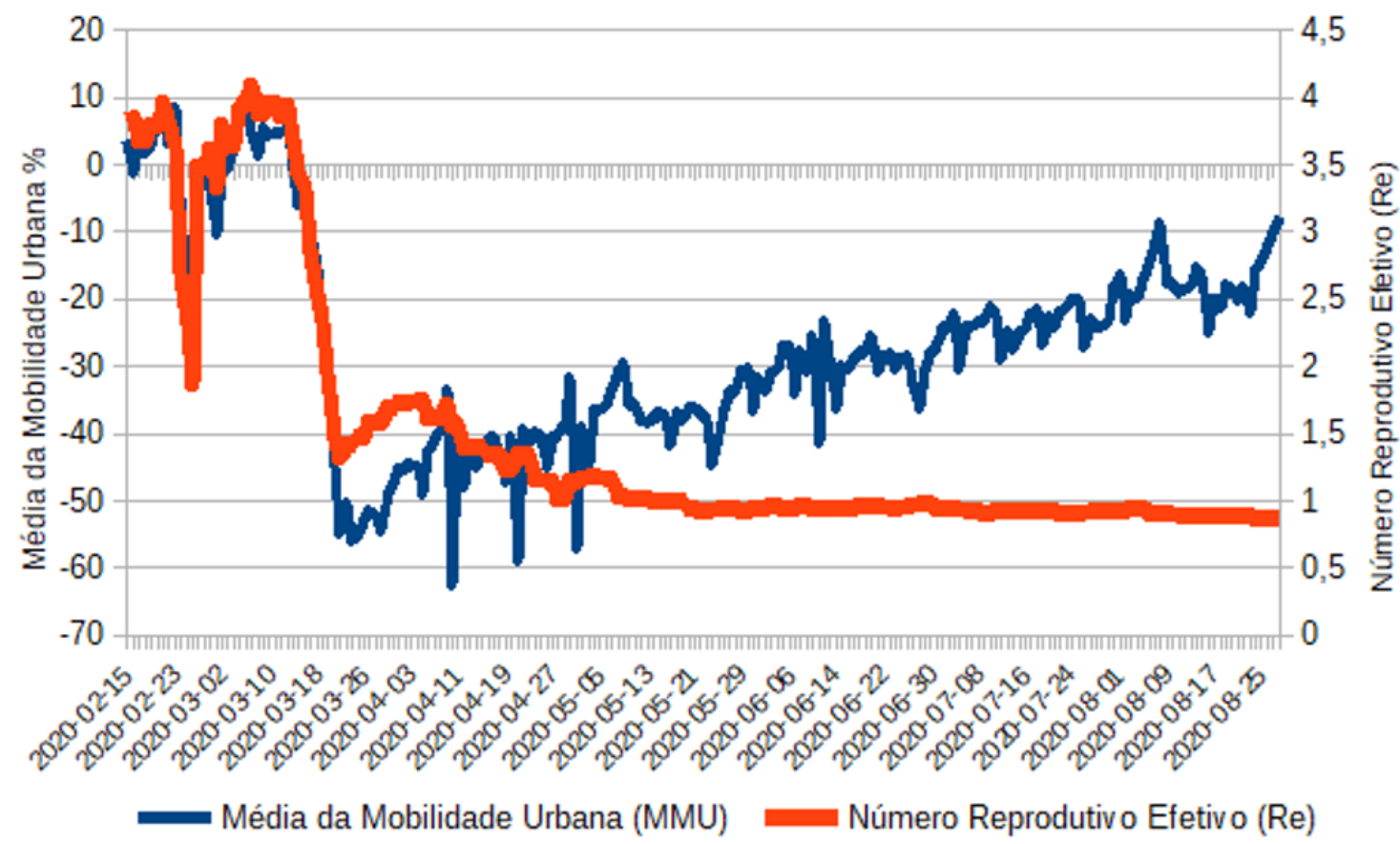

FONTE: BRAZIL AUGUST (2020) e SITUATION REPORT (2020)

\section{DISCUSSÃO}

O modelo SIR é um modelo compartimental que permite modelar a dinâmica de propagação da COVID-19 (JONES, 2019; ISMAIL, 2020). Nesse modelo, o número reprodutivo efetivo $\left(R_{e}\right)$ é uma variável que estima o número de indivíduos que uma pessoa doente irá infectar, em média, ao longo do período de transmissão. Isso é de particular importância pois permite determinar se uma epidemia está em aceleração, estável ou desaceleração. Um $R_{e}>1$ significa que a epidemia está em franca aceleração, enquanto um $R_{e}<1$ significa uma desaceleração, e um $R_{e}=1$ implica em uma epidemia estável. O número reprodutivo básico $\left(R_{0}\right)$, define como o número de indivíduos que serão infectadas por uma pessoa doente, em uma população sem imunidade para a doença, ou seja, em uma situação em que todos são suscetíveis à doença. É possível calcular o $R_{e}$ a partir do Ro utilizando a seguinte fórmula: 


\section{A NC NUCLEO DO conHeCIMENTO ISSN: 2448-0959

\section{$R_{e}=R_{0} \cdot S$}

Em que s é a proporção de suscetíveis dentro da população estudada para a determinada doença.

O Ro, por sua vez, pode ser determinado da seguinte maneira:

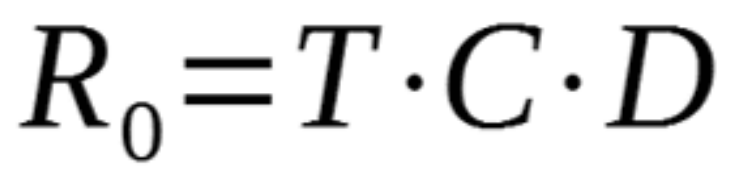

Em que $\mathrm{T}$ é a transmissibilidade (probabilidade de transmissão da doença no contato entre um indivíduo suscetível e um indivíduo doente), C é o número de contatos diários do indivíduo doente, e D é a duração do período transmissível da doença em dias.

Portanto, é possível reformular o número reprodutivo efetivo da seguinte maneira:

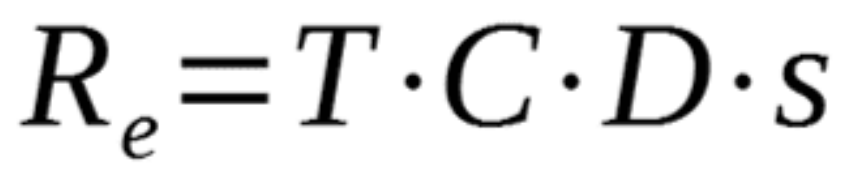

A estabilidade observada no $R_{e}$ no contexto de relaxamento das medidas de distanciamento social no Brasil é verdadeiramente curioso. É plausível inferir que o relaxamento de tais medidas tenha aumentado o contato entre indivíduos com COVID19 e indivíduos suscetíveis (C), que geraria um aumento do Re. Entretanto, tal indicador permaneceu relativamente constante nesse contexto. Assumindo uma duração constante do período de transmissibilidade da COVID-19 em indivíduos doentes (D), a observação de um $R_{e}$ constante pode ser explicada pela redução da transmissibilidade $(\mathrm{T})$ ou pela redução da proporção de suscetíveis (s) na população. 
Uma combinação de ambos fatores provavelmente explica tal achado. A adoção em larga de escala de medidas como o uso de máscaras, interações sociais adaptadas à pandemia e a higiene respiratória no Brasil (DATAFOLHA INSTITUTO DE PESQUISA, 2020) pode também ter gerado uma queda na transmissibilidade (T) da COVID-19 no Brasil. Não há evidência conclusiva na literatura sobre o grau de imunogenicidade do SARS-COV-2, e sobre qual seria a duração e a efetividade dessa imunidade protetora. Portanto, não é possível determinar com precisão qual seria o efeito da propagação da COVID-19 sobre a proporção de suscetíveis (s) na população geral. Em uma situação ideal de indução de imunidade totalmente protetora após a infecção, a Imperial College estima que, isoladamente, tal imunidade já seria responsável por uma diminuição do Re de aproximadamente 30\%. (SITUATION REPORT, 2020)

Existe um terceiro elemento que também poderia justificar a constância do $R_{e}$ no contexto de relaxamento de medidas de distanciamento social. Países como a Coreia do Sul têm demonstrado a efetividade da testagem em massa e da busca ativa e testagem de contatos, permitindo o isolamento de indivíduos infecciosos (SUNA E VIBOUDA, 2020). Apesar do aumento da circulação da população geral nas áreas urbanas brasileiras, a manutenção de baixos níveis de circulação especificamente dos indivíduos com COVID-19 poderia também, em teoria, justificar um $R_{e}$ constante. Entretanto, o baixo número de testes por milhão de habitantes no Brasil sugere que esse componente não exerce um papel tão importante no contexto brasileiro.

O modelo SIR, entretanto, é um modelo relativamente simples com suas limitações. Ao incluir todos os indivíduos infecciosos em um único compartimento, por exemplo, ele não é capaz de estudar separadamente as dinâmicas de portadores sintomáticos da COVID-19, que mais provavelmente serão submetidos a testagem e isolamento domiciliar, e portadores assintomáticos da COVID-19, que dificilmente serão testados $\mathrm{e}$ isolados e provavelmente terão suas atividades inalteradas. O número de contatos diários de cada grupo certamente difere do outro, o que não é levado em consideração nesse modelo epidemiológico. 
A dinâmica de um surto epidêmico é altamente complexa e difícil de ser precisamente prevista. Apesar das epidemias frequentemente serem estudadas no contexto de um país ou qualquer outra área geográfica delimitada, na realidade existem múltiplas pequenas epidemias ocorrendo simultaneamente e em diferentes estágios dentro de um único país, uma única cidade, ou até dentro de um único bairro. Nosso estudo com base no modelo SIR considera o Brasil como um único compartimento homogêneo dentro do qual ocorre o processo epidêmico, que é uma limitação do estudo.

\section{CONCLUSÃO}

A relativa estabilidade do número reprodutivo efetivo da COVID-19 no Brasil entre maio e agosto de 2020 apesar do relaxamento das medidas de distanciamento social observado nesse período pode ser explicado por uma combinação de fatores, conforme o modelo epidemiológico SIR. A redução da transmissibilidade do vírus, dentro do contexto de aumento de uso de máscaras, higiene respiratória e interações sociais adaptadas para o contexto de pandemia; e a redução da proporção de suscetíveis para a doença, decorrente de uma possível imunidade natural, tema ainda controverso na literatura, são fatores que provavelmente justificam a estabilidade do $R_{e}$ no contexto de aumento da circulação em áreas urbanas brasileiras.

\section{REFERENCIAS}

BRAZIL AUGUST. 28, 2020 Mobility changes. COVID-19 Community Mobility Report, 2020. Disponível em: <https://www.gstatic.com/covid19/mobility/2020-0828_BR_Mobility_Report_en.pdf>. Acesso em: 02 de setembro de 2020

ISMAIL, Anas Abou. Modelos Compartimentais da Pandemia COVID-19 para Médicos e Médicos-Cientistas. SN Compr Clin Med. 2020 Jun 4: 1-7.

ISOLAMENTO SOCIAL: MEDO DE SER CONTAMINADO $11 \mathrm{e}$ 12/08/2020. Datafolha Instituto de Pesquisa, 2020. Disponível em: 
<http://media.folha.uol.com.br/datafolha/2020/08/19/34c9d0ae243b593db19bc9652df 8d054reab.pdf> Acesso em: 03 de setembro de 2020

JONES, James Holland. Notes on R. Stanford University, 2019. Disponível em: $<$ http://web.stanford.edu/class/earthsys214/notes/Jones_R0_notes2019.pdf>. Acess o em: 02 de setembro de 2020

SUNA; VIBOUDA. Impacto do rastreamento de contato na transmissão SARS-CoV-

2. Lancet Infect Dis. 2020 Ago; 20(8): 876-877.

SITUATION REPORT FOR COVID-19: Brazil, 2020-09-01. MRC Centre for Global Infectious Disease Analysis, Imperial College London, 2020. Disponível em: $<$ https://mrc-ide.github.io/global-Imic-reports/BRA/>. Acesso em: 02 de setembro de 2020

W. Joost; WIERSINGA, MD, PhD; Andrew Rhodes, MD, PhD; Allen C. Cheng, MD, $\mathrm{PhD} ;$; et al. Fisiopatologia, Transmissão, Diagnóstico e Tratamento da Doença Coronavírus 2019 (COVID-19): Revisão. O JAMA. 2020;324(8):782-793.

Enviado: Setembro, 2020.

Aprovado: Outubro, 2020. 